\title{
The influence of arylene ether positions and phenyl substituents on the physical properties of functional fluorinated polymers
}

\author{
Chun-Che Lee and Wen-Yao Huang
}

We propose four types of poly(arylene ether)s (PAEs) polymers with remarkable thermal stability and high optical transmittance. The PAEs were synthesized by means of nucleophilic displacement through polymerization on a 2-trifluoromethyl-activated bisfluoro monomer to react with bisphenols. Thermal analysis indicated that PAEs possessed a high glass-transition point of $\sim 300{ }^{\circ} \mathrm{C}$ and a decomposition temperature of $T_{\mathrm{d}}=500{ }^{\circ} \mathrm{C}$ at a weight loss of $5 \%$. In addition, a high transmittance of $92 \%$, a low dielectric constant of $\sim 1.9$ and the mechanical properties of PAE films were experimentally determined. The physical properties of PAEs related to the arylene ether positions and phenyl substituents, which showed great potential as substrates for flexible electronics.

Polymer Journal (2011) 43, 180-185; doi:10.1038/pj.2010.116; published online 15 December 2010

Keywords: fluorinated polymer; nucleophilic displacement; thermal stability; morphology; symmetry

\section{INTRODUCTION}

Poly(arylene ether)s (PAEs) are various high-molecular-weight thermoplastics with a twilight zone between amorphous and crystalline. ${ }^{1-6}$ This material has been extensively used because of its excellent mechanical properties, such as high strength capacity, high glasstransition temperature $\left(T_{\mathrm{g}}\right)$, desirable electrical properties, thermooxidative stability, suitable processability, chemical resistance and antihydrolytic characteristics. ${ }^{7-15}$ In general, the PAE polymer is synthesized by the nucleophilic displacement of activated aromatic dihalides and dinitro compounds with alkali-metal bisphenolates, ${ }^{8-11,15}$ which are converted through a Meisenheimer complex. ${ }^{16}$ This nucleophilic displacement method has also been adopted in such commercial engineering plastics as polyethersulfone, ${ }^{13}$ poly(aryl ether sulfone)s, ${ }^{13}$ polyetherimide, ${ }^{2,6}$ poly(aryl ether ketone) $s^{17}$ and polyetheretherketone. ${ }^{18}$ Nucleophilic displacement is an attractive method because of easy synthesis with high yield.

The production of engineering plastics has to fulfill several prerequisites, such as ${ }^{19-21}$ (1) optical transparency in the visible light region; (2) resistance to solvents and stable hydrophobicity; and (3) a high phase-transition temperature $\left(T_{\mathrm{g}}\right.$ higher than $200^{\circ} \mathrm{C}$, continuously useable range of $170-180^{\circ} \mathrm{C}$, melting temperature up to $300^{\circ} \mathrm{C}$ ) and thermal stability to treat with classic manufacturing routes. In addition, the enhancement of tightly packed circuitry has become an increasingly relevant issue that decreases the dielectric constant while maintaining desirable thermal and mechanical properties. ${ }^{22-24}$

Trifluoromethyl $\left(-\mathrm{CF}_{3}\right)$-incorporated polymers are known to have excellent properties, including flame resistance, solubility and thermo-oxidative stability, along with a concomitantly small dielectric constant, water absorption and crystallinity. ${ }^{18,25,26}$ The bulky electronegativity also serves to increase various properties of polymers, including gas permeability and electrical insulation. ${ }^{16}$

There was significant influence on the optical-electronic properties of PAEs because of ring fusing with $\pi$-conjugated and stack framework systems. The thermal stability results from intermolecular interaction, bond length and torsion angle, which also effected to form helical, ladder, lamellar and spherical morphologies. ${ }^{27}$

In this study, we prepared four bis-trifluoromethyl-PAEs that were generated using nucleophilic displacement with different bisphenols. The physical characteristics of PAE films were strongly dependent on the substituents of the bisphenols and the molecular structures. Furthermore, the films showed high thermal stabilities, suitable mechanical properties and consistent transparencies in the visible regime. In this paper, we will describe in detail the synthesis and physical characteristics of PAE polymers.

\section{MATERIALS AND METHODS}

Materials

Commercial reagents and solvents were distilled and deoxygenated under a nitrogen atmosphere before use. Bisphenol monomers are shown in Scheme 1 as A1, A2, B1 and B2, with varying ether positions and phenyl substituents. The polymerization reactions were performed using standard vacuum-line techniques and dehydrated with a slow stream of nitrogen in the reaction vessel throughout the reaction. 


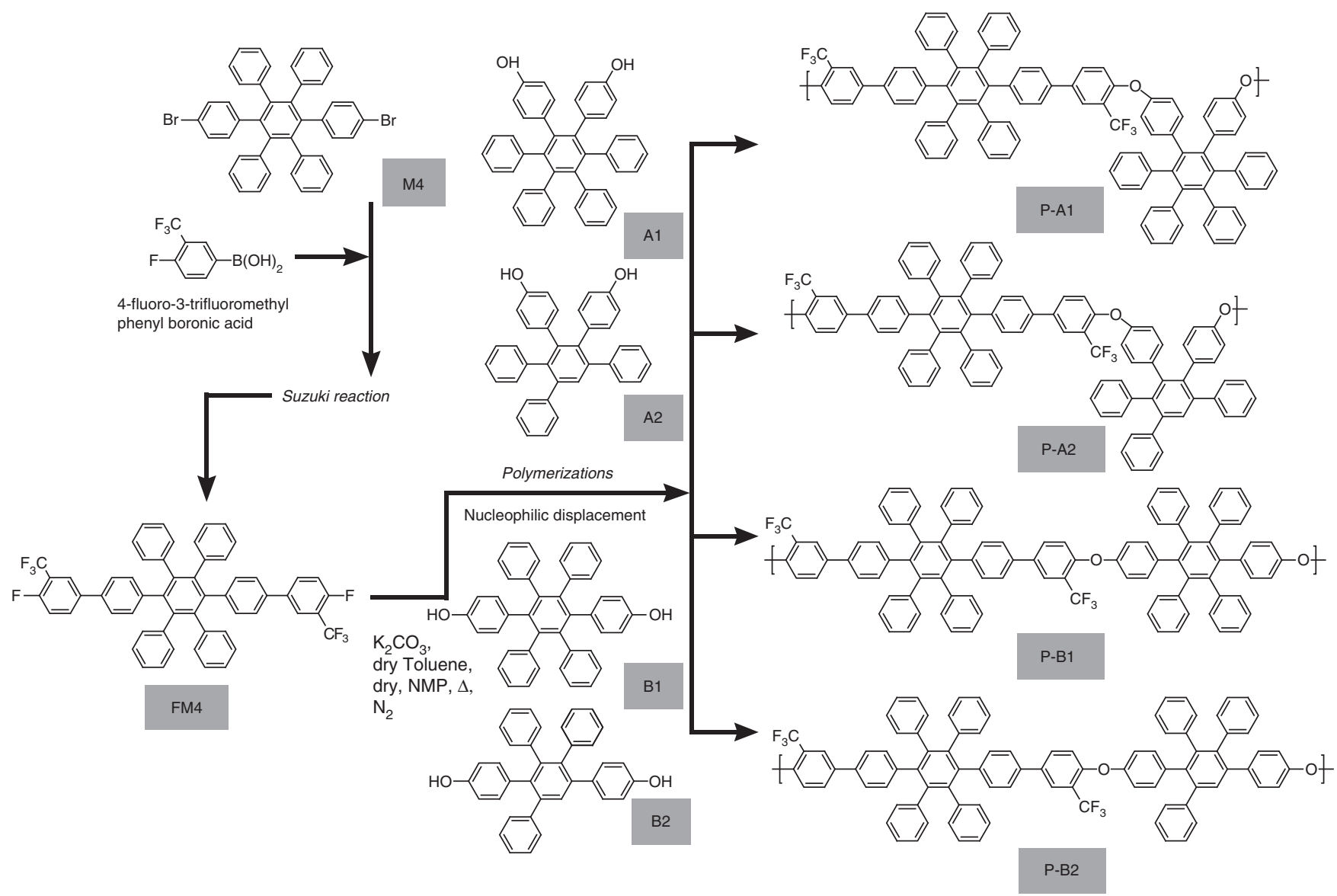

Scheme 1 The synthesis of monomer and PAEs: M4: 4,4"-dibromo-phenylsubstituted $p$-terphenylene. FM4: bis(4-fluoro-3-trifluoromethyl)- $n^{\prime \prime}$-phenylquinquephenyl monomers. A1, A2, B1, B2: substituted bisphenols.

\section{Instrumentation and characterization}

Nuclear magnetic resonance spectra (Varian UNITY INOVA-500 spectrometer, Varian, Sparta, NJ, USA; www.mckscientific.com) were recorded using the solvent chloroform-d with tetramethylsilane as the internal standard. Absorption spectra were recorded with a Perkin Elmer Lambda 35 spectrometer, Perkin Elmer, A\&B Analytical \& Bio Science instruments Co., Ltd, Taipei, Taiwan, ROC. Thermogravimetric analysis (TGA; Hi-Res Perkin Elmer Pyris 1 system, Perkin Elmer) was conducted at a heating rate of $15^{\circ} \mathrm{C}$ per min with a nitrogen stream of $20 \mathrm{~cm}^{3} \mathrm{~min}^{-1}$ to determine the decomposition temperature $\left(T_{\mathrm{d}}{ }^{5 \%}, 5 \%\right.$ of weight loss). All phase-transition temperatures were obtained using a differential scanning calorimeter (Perkin Elmer Pyris Damond DSC (differential scanning calorimeter) system, Perkin Elmer). Gel permeation chromatography analysis was conducted with a Polymer Laboratories HPLC system equipped with GPC KF-803L $(8 \mathrm{~mm}$ i.d. $\times 300 \mathrm{~mm}$; Shodex; Polymer Laboratories, Union, NJ, USA) columns in series using polystyrene as the standard and tetrahydrofuran (THF) as the mobile phase. Varied analysis achieved a precise description of the material properties. Refraction indices $(n)$ were acquired by an ellipsometer (SOPRA GES5, SOPRA Inc., Westford, MA, USA), and surface contact angles were collected using a Drop Shape Analysis System DSA100 (KRÜSS, Oldinburgh Co., Ltd, Taipei, Taiwan). Samples were prepared by spin casting for investigation by atomic force microscopy, scanning electron microscopy and polarized optical microscopy.

The novel 2-perfluoroalkyl-activated bisfluoro monomers were converted into novel PAEs by the nucleophilic displacement of the corresponding activated aromatic dihalo or dinitro compounds with alkali-metal bisphenolates on the benzene ring with bisphenols, as reported in previous studies. ${ }^{1,7,28-31}$ The coupled reactions of six-member ring precursors were performed using the Suzuki reaction ${ }^{27,32}$ with 2.3 equivalents of 4 -fluoro-3-trifluoromethyl phenyl boronic acid in a solvent mixture of toluene, $\mathrm{Na}_{2} \mathrm{CO}_{3(\mathrm{aq})}(1 \mathrm{M})$ and $\mathrm{Pd}\left(\mathrm{PPh}_{3}\right)_{4}$, which were in a reflux apparatus overnight to produce bis(4-fluoro3 -trifluoromethyl)- $n$ "-phenyl-quinquephenyl (FM4) in good yield after purification by silica-gel column chromatography. Polymerization was performed in a Dean-Stark apparatus fitted with a condenser and a nitrogen purge. The flask was charged with FM4 (0.9 mmol), bisphenol (for example, A1, A2, B1 or B2), $\mathrm{K}_{2} \mathrm{CO}_{3}(1.8 \mathrm{~mm}), \quad N$-methyl pyrrolidinone (NMP) $(8 \mathrm{ml})$ and toluene $(15 \mathrm{ml})$. The solution mixture was then stirred at $150{ }^{\circ} \mathrm{C}$ for a few hours, dehydrated in a reaction vessel under a slow stream of nitrogen and then azeotroped with toluene. Subsequently, the resultant mixture was diluted with $8 \mathrm{ml}$ THF and then poured into methanol, stirred and the fibrous polymer precipitated in situ. The precipitated novel PAEs were collected by filtration and washed with methanol several times before vacuum drying until no solvent was detected in the TGA analysis. The yields and molecular polydispersity index $\left(M_{\mathrm{w}} / M_{\mathrm{n}}\right)$ are shown in Table 1. All compounds were purified, and impurities were removed before characterization.

\section{RESULTS AND DISCUSSION}

FM4 were synthesized using a Suzuki coupling reaction between 4,4"-dibromo-phenyl substituted $p$-terphenylene (M4) and 4-fluoro3 -trifluoromethylphenyl boronic acid in good yields. Syntheses were performed using a modification of the published procedure, ${ }^{5,7-10}$ as outlined in Scheme 1. Each FM4 monomer was polymerized with substituted bisphenols of varying degrees to produce four PAEs: P-A1, $\mathrm{P}-\mathrm{A} 2, \mathrm{P}-\mathrm{B} 1$ and P-B2. These illustrations of conformation were distinguished from the linkage of ether $(-\mathrm{O}-)$ between fluorinated monomers and bisphenols. 
Table 1 Thermal and performance characteristics of bisfluoro PAEs

\begin{tabular}{|c|c|c|c|c|c|c|c|c|c|c|c|}
\hline Polymer & $\begin{array}{l}\text { Yield } \\
\text { (\%) }\end{array}$ & $\begin{array}{c}M_{w} \\
\left(\mathrm{~g} \mathrm{~mol}^{-1}\right)\end{array}$ & $\begin{array}{c}M_{n} \\
\left(\mathrm{~g} \mathrm{~mol}^{-1}\right)\end{array}$ & $M_{w} / M_{n}$ & $T_{d}{ }^{5 \%}\left({ }^{\circ} \mathrm{C}\right)$ & $T_{g}\left({ }^{\circ} \mathrm{C}\right)$ & $\begin{array}{c}\text { Contact angle } \\
\text { (water; degrees) }\end{array}$ & $\begin{array}{l}\text { CTE p.p.m. } \\
\text { per }{ }^{\circ} \mathrm{C}\end{array}$ & $\begin{array}{c}\varepsilon_{c a p} \\
(1 \mathrm{kHz})\end{array}$ & $n$ & $\begin{array}{c}\text { Mean of } \\
\text { roughness }(n m)\end{array}$ \\
\hline P-A1 & 81.3 & 391717 & 143301 & 2.73 & 500 & 317 & 135.4 & 23.5 & 1.90 & 1.38 & 2.73 \\
\hline P-B1 & 80.2 & 231617 & 43689 & 5.30 & 548 & 324 & 123.6 & 16.9 & 1.99 & 1.41 & 6.10 \\
\hline P-B2 & 89.3 & 920929 & 219508 & 4.20 & 548 & 318 & 114.3 & 18.8 & 2.01 & 1.42 & 3.27 \\
\hline
\end{tabular}

Abbreviation: CTE, coefficient of thermal expansion.
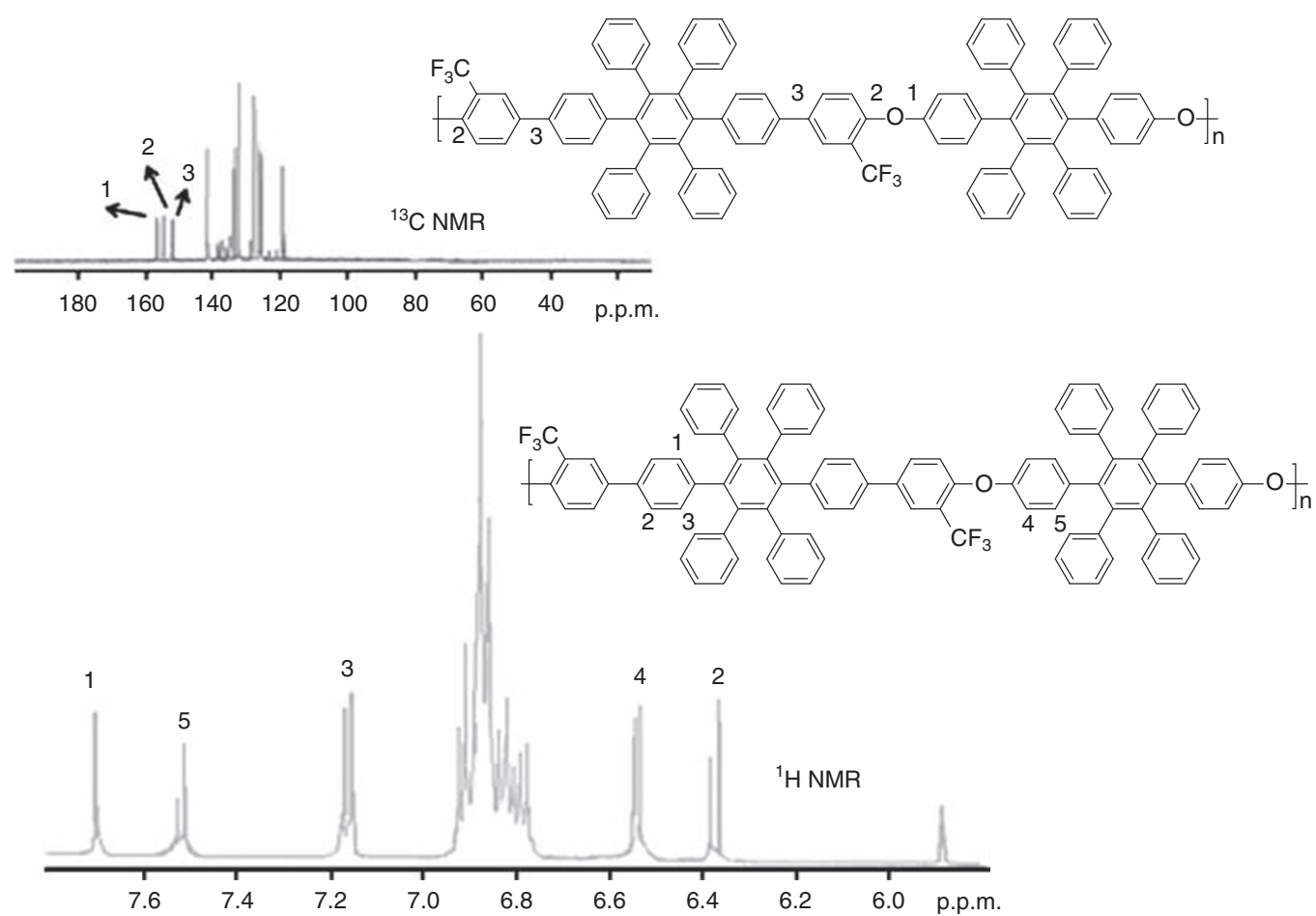

Figure 1 Spectra of ${ }^{1} \mathrm{H}$ and ${ }^{13} \mathrm{C}$ nuclear magnetic resonance (NMR) for $\mathrm{P}-\mathrm{B} 1$ polymer.

The physical properties of the PAE polymer were investigated. These properties were shown to be strongly dependent on the pattern of bisphenol pendants, the monomer structure and the degree of polymer crystallization.

Typical spectroscopic data from ${ }^{1} \mathrm{H}$ and ${ }^{13} \mathrm{C}$ nuclear magnetic resonance spectroscopy are shown in Figure 1 and support the structures of the fluorinated PAEs. The ${ }^{1} \mathrm{H}$ nuclear magnetic resonance spectrum of the P-B1 polymer presents a few peaks around 7.5, 6.6 and 6.4 p.p.m., which were attributed to the trifluoromethyl groups, whereas the signal of chemical shifts caused from the bisfluoro groups of monomer B1 completely disappears because of the nucleophilic displacement of fluorine atoms. The ${ }^{1} \mathrm{H}$ spectra of the polymers do not show any signals corresponding to the terminal $-\mathrm{OH}$ or $-\mathrm{F}$ groups, which is indicative of a high degree of conversion. All results were in good agreement with previous reports. ${ }^{5}$

Compared with the Fourier transform infrared spectroscopy spectrum of PAEs, the disappearance of the foot at around $3500 \mathrm{~cm}^{-1}$ (indicative of fluoro attachment) showed that the nucleophilic displacement of fluorine atoms occurred on the terminal symmetric phenyl ring, which was integrated with bisphenols.

The thermal properties of PAEs were investigated by DSC and TGA. Results revealed the thermal phase transition, decomposition temperature and carbon residue of PAEs in Figures 2 and 3. Data in
Figure 2, which were acquired from DSC analysis, show that the average glass-transition temperature $\left(T_{\mathrm{g}}\right)$ exceeds $300^{\circ} \mathrm{C}$.

The TGA thermogram analyses for PAEs and the monomer (FM4) are shown in Figure 3. Polymers were stable from 450 to $550{ }^{\circ} \mathrm{C}$ in a nitrogen atmosphere. For thermal stability, both FM4 and PAEs could be treated in a high-temperature environment. On the basis of the conformation of the various bisphenols, polymers could be classified into two categories. The first category included P-A1 and P-A2 because they both have a unilateral pendant group with a flexible ether linkage, and they show only one decomposition process that begins at $\sim 550{ }^{\circ} \mathrm{C}$, with char residues of 68 and $75 \%$ at $800{ }^{\circ} \mathrm{C}$, which were attributed to the degradation of the flexible ether leakage and backbone curvature. In contrast to polymers $\mathrm{P}-\mathrm{B} 1$ and $\mathrm{P}-\mathrm{B} 2$ of the second category, these polymers possess phenyl pendant groups attached to the main chain, which indicates two decomposition processes. The first process of weight loss started at about $500{ }^{\circ} \mathrm{C}$, which was attributed to the degradation of benzol, calculated from the proportion of molecular weight loss. On the basis of our previous findings, we know that compared with P-A-type polymers (ortho-bisphenol based), P-B-type polymers (para-bisphenol based) have less thermal stability because of the higher molecular tension in the polymers. The second process that started at $\sim 560{ }^{\circ} \mathrm{C}$ with high char residues could be attributed to the degradation of the backbone chain. 


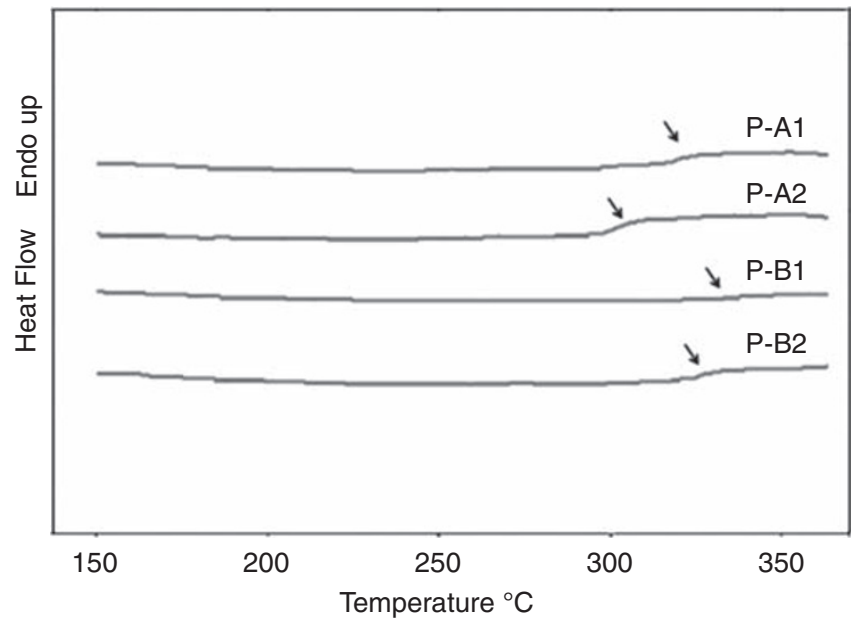

Figure 2 Differential scanning calorimetry curve of PAEs.

The second process started at $\sim 560^{\circ} \mathrm{C}$ with high char residues that could be attributed to the degradation of the backbone chain. Because of the rigid pendant, catenation angle, chain symmetry and intermolecular forces as barriers for free rotation along the main polymer chain, polymers $\mathrm{P}-\mathrm{A} 1$ and $\mathrm{P}-\mathrm{B} 1 \mathrm{had}$ higher $T_{\mathrm{g}}$ values compared with polymers $\mathrm{P}-\mathrm{A} 2$ and $\mathrm{P}-\mathrm{B} 2$, because of the lower degree of rotational freedom along the main chain, as shown in a previous study. ${ }^{33}$

Table 1 summarizes the physical properties of PAE films. The average $T_{\mathrm{g}}$ exceeding $300^{\circ} \mathrm{C}$ presents the outstanding thermal property in TGA analysis. The thermal stability showed that PAEs withstand a high-temperature environment. The highest contact angle of $135.4^{\circ}$ clearly suggested good hydrophobicity. The lower amount of residual water on the polymer surface decreased the chances of possible pollution. In addition, PAEs showed a low coefficient of thermal expansion of around 20 p.p.m. per ${ }^{\circ} \mathrm{C}$, which revealed good mechanical stability. The root mean square (RMS) roughness of $<6 \mathrm{~nm}$ was measured by an atomic force microscope, and demonstrated good planarization. These two mechanical advantages were helpful for the preparation of a stable and smooth substrate. The low dielectric constant ranged from 1.87 to 2.01, as estimated by a Precision Impedance Analyzer (Agilent 4294A, Agilent Technologies Taiwan Ltd, Taipei, Taiwan). Because of these excellent physical properties, the prepared PAEs showed high potential as polymer substrates for high-speed electronic devices. In addition, the good yield and low polydispersity index $\left(1.89<M_{\mathrm{w}} / M_{\mathrm{n}}<5.30\right)$ indicated steady synthesis throughout the experiment. The outstanding mechanical performances are listed in Table 1. The hydrophobic properties showed a $>90^{\circ}$ contact angle on the surface, a refractive index $(n)$ of 1.37-1.42, a low dielectric constant $(k)$ and a value of expansion coefficient (coefficient of thermal expansion) $<30$ p.p.m. per ${ }^{\circ} \mathrm{C}$. In addition, the optical band gap was about $4.5 \mathrm{eV}$, acquired from photoelectron spectroscopy (Riken Keiki Co., Ltd, http://www. rikenkeiki.co.jp/english/contact/).

The photophysical properties of the polymers are shown in Figure 4. Figure 4a shows PAE films and reveals $\pi-\pi^{*}$ transitions with three shoulders at 300, 286 and $256 \mathrm{~nm}$. Both A and B series revealed a geometric effect on adjacent phenylene planes and conjugation lengths. The intensities changed significantly as shown through the altered signal of the ether linkage in the ultraviolet absorption spectra. When comparing P-B1 and P-B2, the effective intermolecular and intramolecular length could be attributed to aggregate formation,

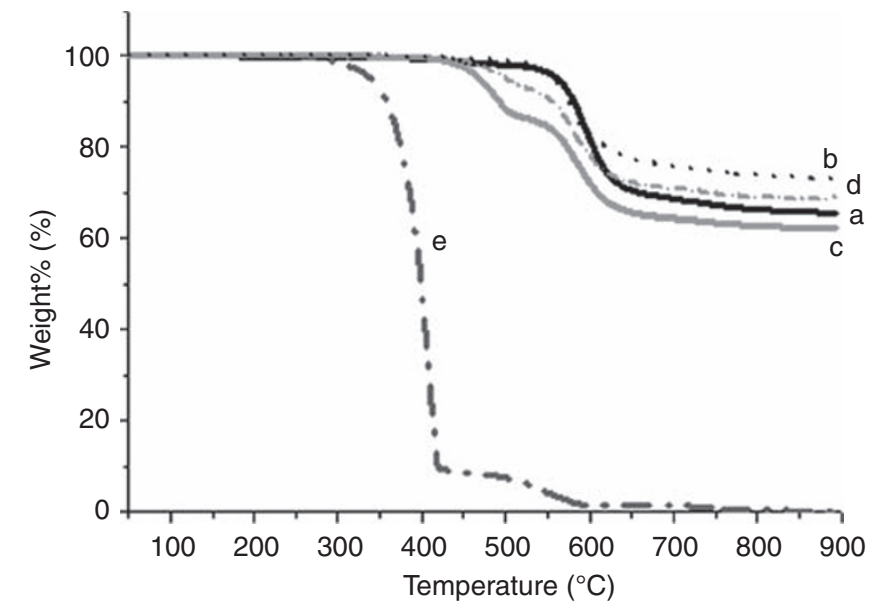

Figure 3 Thermogravimetric analyses of monomer and PAEs. (a) P-A1, (b) P-A2, (c) P-B1, (d) P-B2 and (e) FM4 monomer

which results in an extra absorption band at a longer wavelength with an asymmetric pendant. ${ }^{34}$ Figure $4 \mathrm{~b}$ shows that PAE films conducted no absorption, and also the ultrahigh transmittance of $~ 90 \%$ in the visible light region due to fluorine and various degrees of substituted phenylenes that were relatively free to rotate along the backbone.

The solubility of polymers due to the effects of trifluoromethyl groups and catenation angles is reported in Table 2. PAEs were solubilized in common organic solvents (non-polar and aprotic solvents) at room temperature by sonicating and refluxing. The solvents used were chloroform, toluene, tetrahydrofuran, $N, N^{\prime}$ dimethylformamide (DMF), N,N'-dimethyl acetamide (DMAc) and NMP. A polar protic solvent $(\mathrm{MeOH})$ was tested, but was insoluble and caused swelling.

In appearance, the P-A1 and P-A2 polymers are soft films of easy processability, in contrast to $\mathrm{P}-\mathrm{B} 1$ and $\mathrm{P}-\mathrm{B} 2$, with little hardening, because of the more torsional vibration of the phenyl ring and the similar Kuhn segment motion of pendant groups. Compared with the $\mathrm{A}$ and $\mathrm{B}$ series, $\mathrm{P}-\mathrm{A} 1$ and $\mathrm{P}-\mathrm{B} 1$ polymers provided further proof by DSC of the higher thermal ability of symmetry molecules in the rigid core.

In addition, thermoanalysis revealed an undulant phenomenon at around $150^{\circ} \mathrm{C}$, which implies subtransition evidence of the relationship with flexible and softening behavior.

The verification of morphology and surface chemistry was also shown in the scanning electron microscope and polarized optical microscope (POM) pictures. The molecules squirm into various shapes, which was further visualized using scanning electron microscope. Figures $5 \mathrm{a}$ and $\mathrm{b}$ show that the soft segments appear to form lamellar (folding) and bead shapes with conglomeration on the side view by means of sessile drop preparation. The observations of flat-surface segregation were carried out by spin casting, and the segregation exhibits chain-like (entwined phase) and worm-like dense network surfaces in both P-A1 and P-A2. These formations are assumed to be due to the flexible ether linkage and huge pendant rotation along the backbone, affecting $\pi$-stacking and space filling. Furthermore, in Figures $5 \mathrm{c}$ and d, the linear backbone chain of P-B1 and P-B2 was concerned with symmetry conformation and spatial volume. The blurred spherical conformation and mesoporous morphology in P-B1 is a result of the symmetrical phenylene rings of the main chain and stable space volume twist. The asymmetrical phenylene substituents possess an active vibration and rotation in the 

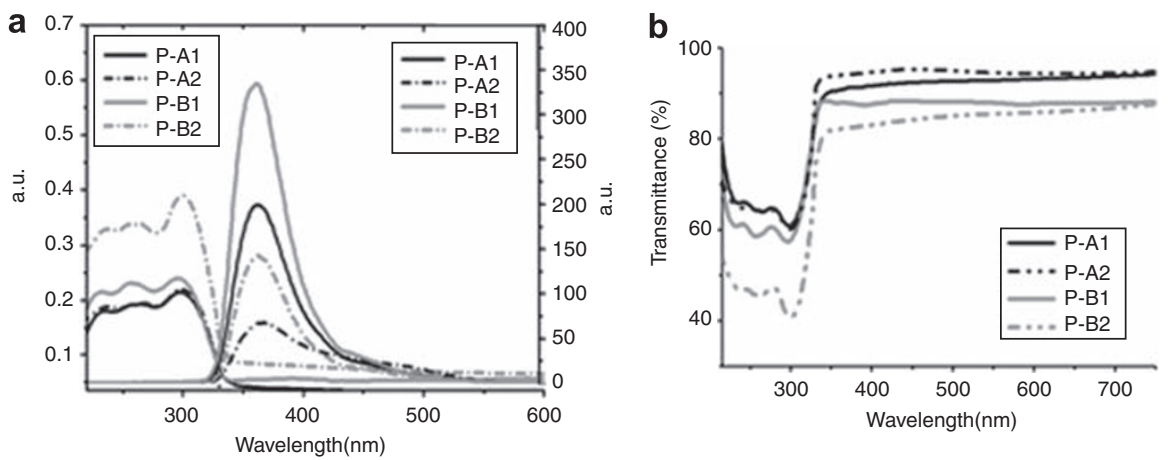

Figure 4 Absorption and photoluminescence spectra of PAEs. (a) Films were prepared by spin casting on quartz using a $1 \%$ concentration in THF. (b) Transmission mode of ultraviolet-visible spectroscopy.

Table 2 Solubilities of bisfluoro poly(arylene ether)s

\begin{tabular}{cccccccc}
\hline Polymer & Methonal & Toluene & $\mathrm{CHCl}_{3}$ & THF & DMF & DMAC & NMP \\
\hline P-A1 & $\times$ & $\odot$ & $\square$ & $\square$ & $\odot$ & $\odot$ & $\odot$ \\
P-A2 & $\times$ & $\odot$ & $\odot$ & $\square$ & $\odot$ & $\odot$ & $\odot$ \\
P-B1 & $\times$ & $\odot$ & $\square$ & $\square$ & $\odot$ & $\odot$ & $\odot$ \\
P-B2 & $\times$ & $\odot$ & $\square$ & $\square$ & $\odot$ & $\odot$ & $\odot$ \\
\hline
\end{tabular}

$\odot$, good; $\square$, general or partial; $\times$, hard.
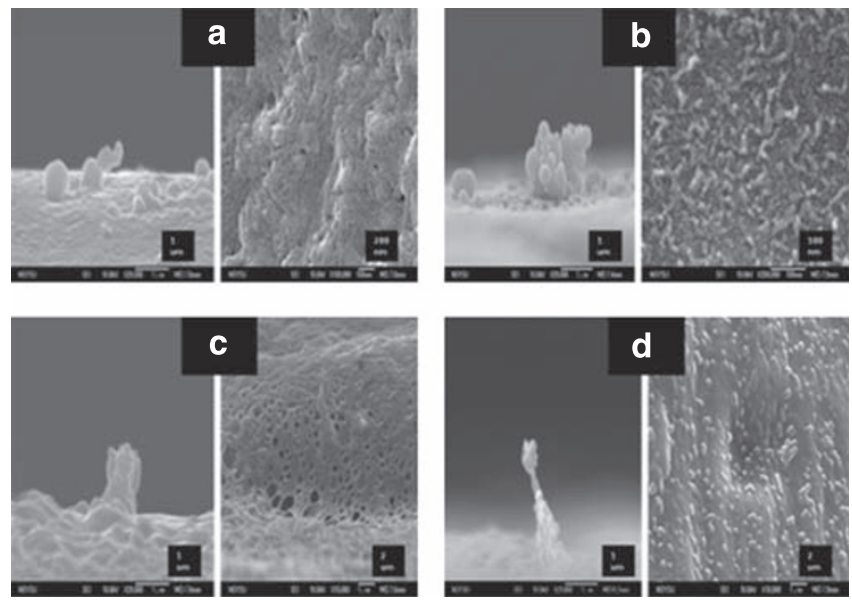

Figure 5 Scanning electron microscope patterns of PAE morphology. Top view (right of letter) and side view (left of letter) patterns that were prepared by solvent casting and shows droplets of dilute. (a) P-A1, (b) P-A2, (c) P-B1 and (d) P-B2.

molecule, which form twists to helical or spherical folding chains derived of two parts, and which easily formed a granular surface. These unique morphologies could be the result of tight chain packing because of the extent of $\pi$-conjugation, the bulky fluorinated group and pendant linkage. In addition, branched/bulky side groups or irregular polymers have longer repeat units and are thus less likely to crystallize, but more likely to be amorphous, as a general rule. Furthermore, amorphous polymers have the advantages of transparency and toughness. For X-ray diffraction techniques, the full-width at half-maximum was measured with a broad range to prove that the structure existed with fewer crystallites, as shown in Supplementary Information. Semicrystalline polymers have advantages due to their chemical resistance and temperature performance. Compared with twisted and beaded shapes, the influence of morphology is described
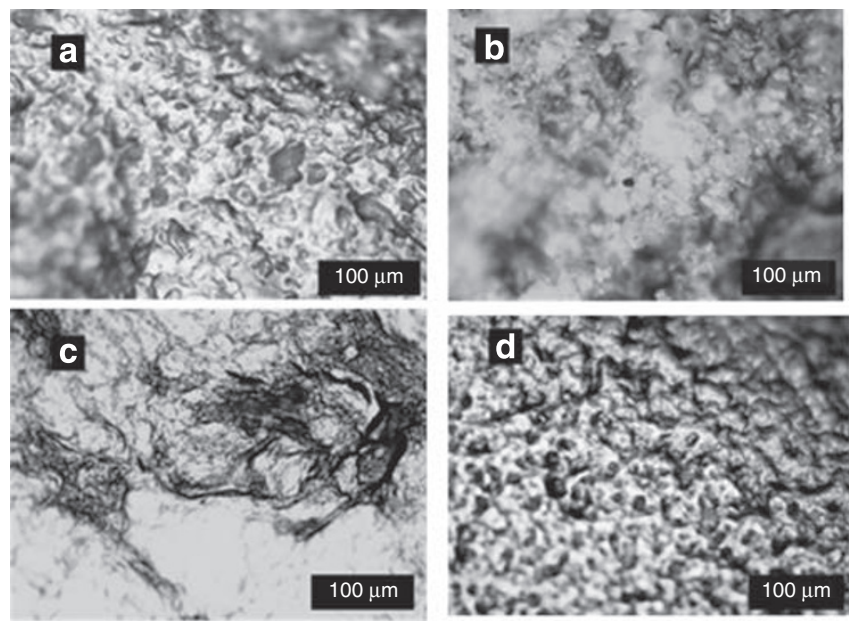

Figure 6 Polarized optical microscopy of PAE films. (a) P-A1, (b) P-A2, (c) P-B1 and (d) P-B2.

in previous research; for example, on thermal plastic and spirolinked molecules. ${ }^{35-39}$

In Figure 6, phase textures were examined by polarized optical microscopy. The effect of pendant size and catenation angle on backbone behavior can be understood in terms of the interactions between neighboring molecules. There are creases and folds along the flat surface in the polymers. We investigated the effect of an asymmetric pendant in P-A2 and P-B2, both as a side-chain and as a main-chain polymer. The fuzzy crystalline particle texture of P-A2 and the clear granule surface of P-B2 are shown in Figures $6 \mathrm{~b}$ and d. These textures indicate that the main and side-chain linkage with the asymmetric pendant that forms various crystallization and aggregation textures on the surface affects the absorption ability conducted in the ultraviolet-visible spectra of the P-B2 polymer. However, in isolated chain emission, all other bands have small Stokes shifts between the emission $\lambda_{\max }$ and the absorption onset, which is characteristic of the aggregation or excimer emission of solid films for conjugated polymers. ${ }^{34}$ The mechanisms of enhanced crystallization and aggregation would be effective in the domains under either thermal annealing or absorption in terms of both bond stretching and distortion of angles between different bonds at a given atom, which is generally interpreted as surface roughness or morphology. For many molecules, however, by far the greater contribution to flexibility stems from rotation about single backbone bonds, which enables the chain to take up a wide range of trajectories. 


\section{CONCLUSION}

Four types of PAEs were prepared as functionally flexible films. The PAE films possessed excellent thermal stabilities $\left(T_{\mathrm{d}}=500{ }^{\circ} \mathrm{C}\right.$ and $T_{\mathrm{g}}=300^{\circ} \mathrm{C}$ ) and remarkable visible light transparencies $(90 \%)$. The physical properties of PAEs were significantly affected by the linking position and the type of bisphenol pendants. Because of the flexible ether linkage $\left(-\mathrm{O}-\right.$ bond angle: $\left.\sim 122^{\circ}\right)$, the bulky $\mathrm{CF}_{3}$ group and molecular conformation, which included bond length, torsion angle and packing density ${ }^{40}$ showed a wide range of functions. The properties encompassed the following: increased solubility, chemical resistance, glass-transition temperature, thermal stability, oxidation resistance, decreased color and intermolecular charge transfer complex (but high optical transparency of $\sim 90 \%$ ), crystallinity, dielectric constant and water absorption. Because of the excellent physical advantages, the prepared PAEs are good candidates for flexible electronics.

\section{SUMMARY}

These fluorinated poly(aryl ether)s with altered ether positions of phenyl substituents provide excellent mechanical properties, such as high-level strength and high glass-transition temperature, good electrical properties, thermo-oxidative stability, good processability and chemical resistance, and they are stably antihydrolytic. Finally, we applied PAE films as flexible subtracts to deposit indium tin oxide (thickness $\sim 150 \mathrm{~nm}$ ); the preliminary consequence of the sheet resistance is below $30 \Omega$ per ${ }^{\circ} \mathrm{C}$ at room temperature.

1 Bottino, F. A., Pasquale, G.Di. \& lannelli, P. Synthesis, characterization, and study of the thermal properties of new poly(arylene ether 134-oxadiazole)s. Macromolecules 34, 33-37 (2001)

2 Hedrick, J. L. Poly (aryl ether amides). Macromolecules 24, 812-813 (1991).

3 Huang, W. Y., Liaw, B. R., Chang, M. Y., Han, Y. K. \& Huang, P. T. High glass-transition temperature and organosoluble novel arylene ether polymers. Macromolecules 40, 8649-8657 (2007).

4 Chikashige, Y., Chikyu, Y., Miyatake, K. \& Watanabe, M. Poly (arylene ether) ionomers containing sulfofluorenyl groups for fuel cell applications. Macromolecules $\mathbf{3 8}$, 7121-7126 (2005).

5 Liaw, B. R., Huang, W. Y., Huang, P. T., Chang, M. Y. \& Han, Y. K. Novel poly(arylene ether)s containing multi-substituted pentaphenylene moiety. Polymer 48, 7087-7097 (2007).

6 Hedrick, J. L. \& Labadie, J. W. Poly (aryl ether phenylquinoxalines). Macromolecules 23, 1561 (1990).

7 Xu, Z., Dannenberg, C., Springer, J., Banerjee, S. \& Maier, G. Gas separation properties of polymers containing fluorene moieties. Chem. Mater. 14, 3271-3276 (2002).

8 Bourgeois, Y., Charlier, Y., Devaux, J. \& Legras, R. Synthesis of a poly(aryl ether sulfone)-poly(aryl ether ketone) triblock copolymer. Polymer 37, 5503-5511 (1996).

9 Banerjee, S. \& Maier, G. Novel high Tg high-strength poly(aryl ether)s. Chem. Mater. 11, 2179-2184 (1999).

10 Chikashige, Y., Chikyu, Y., Miyatake, K. \& Watanabe, M. Poly (arylene ether) ionomers containing sulfofluorenyl groups for fuel cell applications. Macromolecules $\mathbf{3 8}$ 7121-7126 (2005)

11 Qi, Y., Ding, J., Day, M., Jiang, J. \& Callender, C. L. Cross-linkable highly fluorinated poly(arylene ether ketones/sulfones) for optical waveguiding applications. Chem. Mater. 17, 676-682 (2005).

12 Aitken, C. L., McHattie, J. S. \& Paul, D. R. Dynamic mechanical behavior of polysulfones. Macromolecules 25, 2910-2922 (1992).

13 Attwood, T. E., Barr, D. A., Feasey, G. G., Leslie, V. J., Newton, A. B. \& Rose, J. B. Poly(arylene ether sulphones) by polyetherification: 1 synthesis of halogenophenols. Polymer 18, 354-358 (1997).

14 Palmans Anja, R. A., Smith, P. \& Weder, C. Polarizing energy transfer in photoluminescent conjugated polymers with covalently attached sensitizers. Macromolecules 32, 4677-4685 (1999).
15 Tullos, G. L. \& Cassidy, P. E. Polymers derived from hexafluoroacetone: 12F-Poly(ether ketone). Macromolecules 24, 6059-6064 (1991).

16 Saran, N., Parikh, K., Suh, D. S., Muñoz, E., Kolla, H. \& Manohar, S. K. Fabrication and characterization of thin films of single-walled carbon nanotube bundles on flexible plastic substrates. J. Am. Chem. Soc. 126, 4462-4463 (2004).

17 Tullos, G. L., Cassidy, P. E. \& St Clair, A. K. Polymers derived from hexafluoroacetone: 12F-poly (ether ketone). Macromolecules 24, 6059-6064 (1991).

18 Park, S. K. \& Kim, S. Y. Synthesis of poly(arylene ether ketone)s containing trifluoromethyl groups via nitro displacement reaction. Macromolecules 31, 3385-3387 (1998).

19 Liu, P., Wu, Y., Li, Y., Ong, B. S. \& Zhu, S. Enabling gate dielectric design for all solution-processed high-performance flexible organic thin-film transistors. J. Am. Chem. Soc. 128, 4554-4555 (2006).

20 Zhao, G., Ishizaka, T., Kasai, H., Oikawa, H. \& Nakanishi, H. Fabrication of unique porous polyimide nanoparticles using a reprecipitation method. Chem. Mater. 19, 1901-1905 (2007)

21 Su, K., Bujalski, D. R., Eguchi, K., Gordon, G. V., Ou, D. L., Chevalier, P., Hu, S. \& Boisvert, R. P. Low-k interlayer dielectric materials: synthesis and properties of alkoxyfunctional silsesquioxanes. Chem. Mater. 17, 2520-2529 (2005).

22 US patent no. 4835197, 5658994, 5108840, 5114780, 5145936 and 5155175.

23 Lu, Z., Shao, P., Li, J., Hua, J., Qin, J., Qin, A. \& Ye, C. Two novel fluorinated poly(arylene ether)s with pendant chromophores for second-order nonlinear optical application. Macromolecules 37, 7089-7096 (2004).

24 Ding, J., Du, X., Day, M., Jiang, J., Callender, C. L. \& Stupak, J. Highly fluorinated poly(arylene alkylene ether sulfone)s: synthesis and thermal properties. Macromolecules 40, 3145-3153 (2007).

25 Ganapathy, H. S., Hwang, H. S. \& Lim, K. T. Synthesis and properties of fluorinated ester-functionalized polythiophenes in supercritical carbon dioxide. Ind. Eng. Chem. Res. 45, 3406-3411 (2006).

26 Hill, D. J., Mio, M. J., Prince, R. B., Hughes, T. S. \& Moore, J. S. A field guide to foldamers. Chem. Rev. 101, 3893-4011 (2001).

27 Huang, C., Zhen, C. G., Su, S. P., Loh, K. P. \& Chen, Z. K. Solution-processable polyphenylphenyl dendron bearing molecules for highly efficient blue light-emitting diodes. Org. Lett. 7, 391-394 (2005).

28 Johnson, B. C., Yilgör, I.., Tran, C., Iqbal, M., Wightman, J. P., Lloyd, D. R. \& McGrath, J. E. Synthesis and characterization of sulfonated poly(acrylene ether sulfones). J. Polym. Sci. Polym. Chem. Ed. 22, 721-737 (1984)

29 Pixton, M. R. \& Paul, D. R. Gas transport properties of adamantane-based polysulfones. Polymer 36, 3165-3172 (1995).

30 Bottino, F. A., Pasquale, G.Di. \& lannelli, P. Synthesis characterization and study of the thermal properties of new poly(arylene ether 134-oxadiazole)s. Macromolecules 34, 33-37 (2001)

31 Wolfe, J. F. \& Arnold, F. E. Rigid-rod polymers 1 synthesis and thermal properties of para-aromatic polymers with 26-benzobisoxazole units in the main chain. Macromolecules 14, 909-915 (1981).

32 Huang, W. Y., Gao, W., Kwei, T. K. \& Okamoto, Y. Synthesis and characterization of poly(alkyl-substituted p-phenylene ethynylene)s. Macromolecules 34, 1570-1578 (2001).

$33 \mathrm{McHattie}$, J. S., Koros, W. J. \& Paul, D. R. Effect of isopropylidene replacement on gas transport properties of polycarbonates. J. Polym. Sci. B Polym. Phys. 29, 731-746 (1991).

34 Huang, W. Y., Matsuoka, S., Kwei, T. K. \& Okamoto, Y. Aggregation and gelation of fully conjugated rigid-rod polymers poly(2,5-dialkyl-1,4-phenyleneethynylene)s. Macromolecules 34, 7166-7171 (2001).

35 Hansen, E. F., Derrick, M. R., Schilling, M. R. \& Garcia, R. The effects of solution application on some mechanical and physical properties of thermoplastic amorphous polymers used in conservation: poly(vinyl acetate)s. J. Am. Inst. Conser. 30, 203-213 (1991).

36 Ikeda, K., Ogoma, Y., Fujii, T., Hachimori, A., Kondo, Y., Hayakawaa, T., Iwatsuki, M. \& Akaike, T. Conformation of polyamino acids containing fluorine. Polymer 31, 344-347 (1990).

37 Lee, S. H., Jang, B. B. \& Kafafi, Z. H. Highly fluorescent solid-state asymmetric spirosilabifluorene derivatives. J. Am. Chem. Soc. 127, 9071-9078 (2005).

38 Ohshita, J., Lee, K. H., Hamamoto, D., Kunugi, Y., Ikadai 1, J., Kwak, Y. W. \& Kunai, A. Synthesis of novel spiro-condensed dithienosiloles and the application to organic FET. Chem. Lett. 33, 892-893 (2004).

39 Jang, W., Shin, D., Choi, S., Park, S. \& Han, H. Effects of internal linkage groups of fluorinated diamine on the optical and dielectric properties of polyimide thin films. Polymer 48, 2130-2143 (2007).

40 Colquhoun, H. M. \& Williams, D. J. Crystal and molecular simulation of highperformance polymers. Acc. Chem. Res. 33, 189-198 (2000).

Supplementary Information accompanies the paper on Polymer Journal website (http://www.nature.com/pj) 